\title{
Nueva Estrategia de Control para la Reducción de Oscilaciones en Turbinas Eólicas Flotantes
}

\author{
Payam Aboutalebi, Fares M’zoughi, Izaskun Garrido, Aitor J. Garrido
}

Grupo de Control Automático (ACG). Instituto de Investigación y Desarrollo de Procesos. Escuela de Ingeniería de Bilbao. Universidad del País Vasco/Euskal Herriko Unibertsitatea (UPV / EHU). P Pafael Moreno, 3. 48013 Bilbao. España

payam.aboutalebi@ehu.eus; fares.mzoughi@ehu.eus; izaskun.garrido@ehu.eus; aitor.garrido@ehu.eus

\begin{abstract}
Resumen
En este trabajo se ha propuesto una nueva estrategia de control para mejorar la estabilidad de turbinas eólicas flotantes (FWT). Al objeto de estudiar la reducción de oscilaciones no deseadas en el sistema, y en particular en los movimientos de cabeceo de la plataforma y de proa-popa de la nacelle, se ha considerado una plataforma tipo barcaza de forma cuadrada y equipada con cuatro columnas de agua oscilantes (OWC) colocadas de manera simétrica. De esta forma, las válvulas de control de flujo de aire de las cámaras de captura permiten operar las citadas columnas de aire para controlar los movimientos de la barcaza causados por la dinámica oscilatoria de la ola incidente. Para ello, se ha efectuado un análisis de los operadores de amplitud de respuesta (RAO) que permite implementar una nueva estrategia de control de conmutación para regular adecuadamente la transición apertura/cierre de las válvulas de control de flujo. Los resultados obtenidos muestran que la topología de plataforma híbrida propuesta, dotada de sistemas controlados OWC, presenta un mejor rendimiento que una plataforma análoga tradicional.
\end{abstract}

Palabras clave: turbina eólica flotante; columna de agua oscilante; energía de olas; energía eólica; estabilización; operador de amplitud de respuesta; control de conmutación

\section{Introducción}

Los recursos sostenibles de las energías renovables, como la energía eólica y de las olas, están atrayendo cada vez más atención debido al cambio climático y los impactos del calentamiento global. Para luchar contra estos problemas, las turbinas eólicas flotantes están llamadas a desempeñar un papel sin precedentes en la captura de recursos energéticos renovables, permitiendo una mayor penetración en el mix energético de este tipo de energías limpias.

La estructura de las FWT ofrece la posibilidad de añadir columnas OWC [1], que compone el tipo mejor conocido de convertidores de energía de las olas [2], para aprovechar simultáneamente el recurso eólico y undimotriz. No obstante, una de las ventajas de considerar ambos tipos de dispositivos en una misma plataforma es la posibilidad de utilizar las plantas OWC no solo para producir energía, sino como elemento activo que permita estabilizar la plataforma, evitando rangos de vibración perjudiciales. En este sentido, el objetivo es doble: Estabilizar la FWT aliviando vibraciones no deseadas y generar la máxima energía posible [3]. Estas vibraciones no deseadas reducen la eficiencia aerodinámica, acortan la vida de fatiga de la torre debida a la fatiga de sus materiales y aumentan las tensiones en las palas, el eje del rotor, el cojinete de guiñada y la misma base de la torre [4]. Por tanto, es importante restringir los movimientos oscilatorios de la plataforma a un rango adecuado que evite estos efectos aumentando el rendimiento y en última instancia, el LCoE.

Del estudio del estado del arte del problema, se puede observar que diversos autores han aplicado distintos métodos y estrategias el fin de limitar los movimientos de las FWTs [5]. Por ejemplo, M. Kamarlouei y col. en [6] llevó a cabo un estudio experimental para reducir la amplitud de cabeceo y elevación utilizando cables de amarre de catenaria añadidos, así como la instalación de convertidores de energía de las olas (WEC) en un FWT. Asimismo, varios autores han propuesto el uso de dispositivos pasivos de control tipo inercial sobre plataformas FWT al objeto de controlar los desplazamientos de la torre (ver Y. Zhang et al. en [7] y Y. Hu et al. en [8]).

Menos artículos han ampliado la aplicación de FWTOWC combinados basados en barcazas con la consideración de ecuaciones de movimiento no lineales completas. En este sentido, J.M. Jonkman 
([9]) diseñó una piscina de forma cuadrada ubicada en el centro de la barcaza que ofrece la opción de agregar un dispositivo OWC. No obstante, este sistema híbrido no se ha diseñado con fines de estabilización y no permite tal estudio. Por su parte, P. Aboutalebi et al. ([10]) analizó el rendimiento de sistemas híbridos FWT-OWC tipo barcaza para diferentes estados de la mar con el fin de incrementar la estabilidad de la plataforma. Sin embargo, ninguno de los enfoques introducidos ha propuesto hasta ahora una estrategia de control de las válvulas OWC.

Así, en este artículo se utiliza un modelo no lineal basado en la topología NREL 5-MW tipo barcaza, alojando cuatro sistemas OWC en la misma con el fin de disminuir las oscilaciones, particularmente en los modos de cabeceo y de proa a popa de la torre, en varios estados de la mar. Además, haciendo uso de los operadores de amplitud de respuesta, se ha desarrollado una la estrategia de control de conmutación para la regulación de las válvulas de control de flujo de los OWC. De esta forma, se ha pidi comparar el sistema FWT basado en OWC controlado con el análogo tradicional tipo barcaza para mostrar la bondad de la topología híbrida propuesta.

El resto del artículo está estructurado como sigue: La Sección 2 explica la estructura del sistema híbrido FWT-OWC a partir de sus ecuaciones dinámicas. La Sección 3 estudia el problema y desarrolla los RAOs utilizados en la estrategia de control de conmutación propuesta. La sección 4 presenta analiza la eficiencia del control de conmutación propuesto para diferentes estados de la mar. Y finalmente, en la Sección 5, se presentan las conclusiones oportunas a partir de los resultados obtenidos.

\section{Descripción del modelo}

\subsection{Descripción de FWT}

Se ha considerado la topología de plataforma FWT híbrida OWC representada en la Figura 1. La plataforma está lastrada mediante inundación al objeto de obtener el calado adecuado no vulnerable a golpe de onda continua. La plataforma además se ancla mediante ocho cables de catenaria, con dos de las líneas de esquina de la parte inferior a un ángulo de 45 grados.

Se evalúan ocho grados de libertad (DOF, por sus siglas en inglés), a saber, desplazamientos en el plano, elevación, cabeceo, balanceo, guiñada y desplazamientos babor-estribor y proa-popa de la torre, con el origen del sistema de coordenadas en la línea de flotación. En la Tabla 1 se presentan las características detalladas de la turbina eólica.

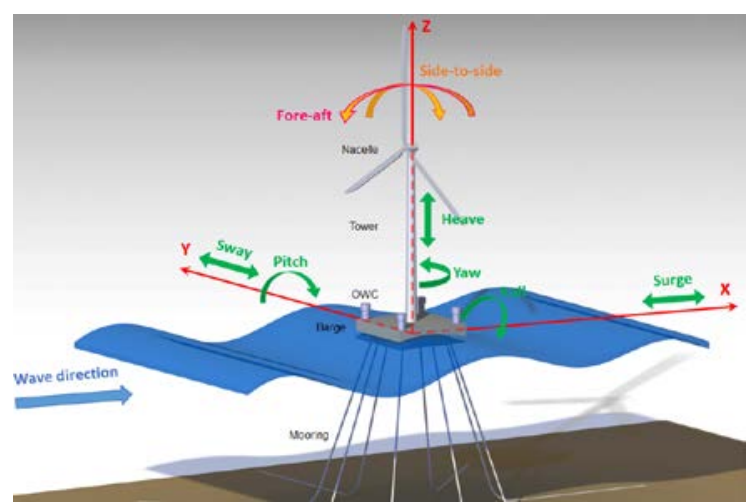

Figura 1. Turbina eólica marina flotante basada en barcazas con cuatro OWC

Tabla I: Características de la barcaza ITI

\begin{tabular}{|c|c|}
\hline Parámetro & Valor \\
\hline $\begin{array}{l}\text { Dimensiones plataforma }(\mathrm{W} \times \\
\mathrm{L} \times \mathrm{H})\end{array}$ & $40 \mathrm{~m} \times 40 \mathrm{~m} \times 10 \mathrm{~m}$ \\
\hline $\begin{array}{l}\text { Dimensiones OWC }(\mathrm{W} \times \mathrm{L} \times \\
\mathrm{H})\end{array}$ & $5 \mathrm{~m} \times 5 \mathrm{~m} \times 10 \mathrm{~m}$ \\
\hline Calado & $4 \mathrm{~m}$ \\
\hline $\begin{array}{l}\text { Desplazamiento de agua } \\
\text { barcaza estándar }\end{array}$ & $6400 \mathrm{~m} 3$ \\
\hline $\begin{array}{l}\text { Desplazamiento de agua para } \\
\text { barcaza OWC }\end{array}$ & $6000 \mathrm{~m} 3$ \\
\hline Masa, incluido lastre & $5,452,000 \mathrm{~kg}$ \\
\hline CM-Ubicación bajo SWL & $0.281768 \mathrm{~m}$ \\
\hline Inercia balanceo sobre CM & $726,900,000 \mathrm{~kg} \cdot \mathrm{m} 2$ \\
\hline Inercia cabeceo sobre CM & $726,900,000 \mathrm{~kg} \cdot \mathrm{m} 2$ \\
\hline Inercia guiñada sobre CM & 1,453,900,000 kg.m2 \\
\hline Profundidad del ancla (agua) & $150 \mathrm{~m}$ \\
\hline $\begin{array}{l}\text { Separación entre anclas } \\
\text { opuestas }\end{array}$ & $773.8 \mathrm{~m}$ \\
\hline $\begin{array}{l}\text { Longitud de la línea sin } \\
\text { tensionar }\end{array}$ & $473.3 \mathrm{~m}$ \\
\hline $\begin{array}{l}\text { Longitud de línea neutra sobre } \\
\text { lecho marino }\end{array}$ & $250 \mathrm{~m}$ \\
\hline Diámetro de la línea & $0.0809 \mathrm{~m}$ \\
\hline Densidad de masa línea & $130.4 \mathrm{~kg} / \mathrm{m}$ \\
\hline Rigidez línea & $589,000,000 \mathrm{~N}$ \\
\hline
\end{tabular}

Se han considerado las ecuaciones dinámicas para una turbina eólica marina de $5 \mathrm{MW}$ instalada en la plataforma híbrida descrita. Las respuestas dinámicas de la FWT se han introducido en FAST al objeto de obtener las correspondientes ecuaciones de movimiento no lineales.

Así, las ecuaciones no lineales completas del movimiento en el dominio del tiempo de la turbina eólica flotante acoplada, el sistema de plataforma de soporte y los OWC son de la forma: 


$$
M_{i j}(q, u, t) \ddot{q}_{j}=f_{i}(q, \dot{q}, u, t)
$$

donde $M_{i j}$ es el componente $(i, j)$ de la matriz de masa de inercia, $u$ son las entradas de control, $t$ es el tiempo, $\ddot{q}_{j}$ es la segunda derivada del tiempo del $j$-ésimo DOF y $f_{i}$ es el componente de la función de fuerza asociada al DOFi, $f_{i}$ es la fuerza exterior generalizada, incluida la carga aerodinámica sobre las palas y la góndola, la fuerza hidrodinámica sobre la plataforma, las fuerzas elásticas y servo.

Por tanto, el sistema de ecuaciones lineales de movimiento en el dominio de la frecuencia se puede escribir como:

$I_{F W T}(\omega) \ddot{q}+B_{F W T}(\omega) \dot{q}+C_{F W T} \vec{q}=\vec{f}_{F W T}(\omega)$

donde $I_{F W T}, B_{F W T}$ y $C_{F W T}$ son las matrices de inercia, amortiguación y rigidez respectivamente, y $q$ se define como:

$$
q=\left[\begin{array}{c}
\text { surge } \\
\text { sway } \\
\text { heave } \\
\text { roll } \\
\text { pitch } \\
\text { yaw } \\
\text { fore }- \text { aft } \\
\text { side }- \text { to }- \text { side }
\end{array}\right]
$$

La matriz de inercia a su vez viene definida como:

$$
I_{\text {FOWT }}(\omega)=A_{\text {Hydro }}(\omega)+M_{\text {Platform }}+M_{\text {Tower }}
$$

donde $M_{\text {Platform }}$ y $M_{\text {Tower }}$ son las matrices de masa de plataforma y torre, respectivamente. El acoplamiento de los elementos de los modos de flexión de la torre con la plataforma se integra en la matriz $M_{\text {Tower }}$. Y por último $A_{H y d r o}(\omega)$ es la masa añadida de la plataforma y OWC calculada a través de WAMIT a partir del análisis de radiación del panel y depende de la frecuencia. La ecuación (3) muestra los modos del sistema con los seis primeros para los movimientos de la plataforma y los dos últimos para los desplazamientos de flexión de la torre.

Por su parte, la matriz de amortiguación se define como:

$$
B_{F W T}(\omega)=B_{\text {Hydro }}(\omega)+B_{\text {Tower }}+B_{\text {viscous }},
$$

donde $B_{H y d r o}(\omega)$ es la matriz de amortiguación de la plataforma flotante y OWC que se obtiene mediante WAMIT del análisis de radiación de panel, $B_{\text {Tower }}$ es la matriz de amortiguación de la torre flexible y $B_{\text {viscous }}$ representa el arrastre viscoso no lineal en la plataforma.

Asimismo, la matriz de rigidez se define como:

$$
C_{F W T}=C_{\text {Hydro }}+C_{\text {Mooring }}+C_{\text {Tower }}
$$

donde $C_{\text {Hydro }}$ se obtiene mediante WAMIT a partir de la matriz de restauración hidrostática de la plataforma y los OWC, $C_{\text {Mooring }}$ contiene los coeficientes de rigidez del resorte de las líneas de amarre y $C_{\text {Tower }}$ representa la matriz de rigidez de la torre.

Por último, el término del lado derecho de la ecuación (2) viene dado por:

$$
\vec{f}_{F W T}(\omega)=\vec{f}_{\text {Hydro }}(\omega)+\vec{f}_{\text {vis c ous }}
$$

donde $\vec{f}_{\text {viscous }}(\omega)$ es la fuerza viscosa, $\vec{f}_{\text {Hydro }}(\omega)$ representa la fuerza hidrodinámica de las olas en la plataforma y $\vec{f}_{\text {Hydro }}(\omega)$ se obtiene mediante el análisis de difracción de panel en WAMIT.

\subsection{Diseño y cálculo de la plataforma}

Mediante el paquete de computación MultiSurf se han diseñado y comparado tres topologías de plataforma diferentes a utilizar posteriormente: Una primera denominada barcaza estándar de forma cuadrada como se muestra en la Figura 2a. Un segundo modelo consistente en una plataforma de barcaza basada en OWC con cuatro dispositivos alojados en las esquinas con válvulas de control de flujo de aire cerradas, como se ilustra en la Figura 2b. La Figura 2c por su parte muestra el tercer modelo, consistente en una plataforma con cuatro dispositivos OWC con válvulas de control de fujo abiertas.

\section{Planteamiento del problema}

Tal como se ha indicado, las oscilaciones en los FWT no son deseables ya que tales movimientos deseados tienen efectos negativos en el sistema, incluida la tensión en los componentes estructurales del sistema y 
una reducción drástica de la capacidad de captación de energía eólica y undimotriz. Así como un incremento de los costos de mantenimiento y consiguiente reducción del LCoE. Para hacer frente a este problema, debe analizarse con precisión el comportamiento del sistema frente a diferentes estados de la mar.

\subsection{Análisis de la dinámica de FWTs mediante operadores de amplitud de respuesta}

La utilización de RAO es fundamental para la evaluación del movimiento de sistemas marinos en diferentes escenarios y en particular para en análisis de la dinámica de las FWTs. En esta sección, se presenta el procedimiento utilizado para establecer los RAO para diferentes modos del sistema de entradasalida en el dominio de la frecuencia, para los estados de traslación y rotación del mismo. Primeramente, se emplean los datos geométricos de la plataforma de barcaza estándar, la plataforma de barcaza basada en OWCs abiertas y la plataforma de barcaza basada en OWCs cerradas para realizar los cálculos de las matrices hidrodinámicas, hidrostáticas, de amortiguación y de masa agregada mediante WAMIT. Las matrices obtenidas se introducen entonces en las ecuaciones de movimiento no lineales con el objetivo de obtener los resultados de cada uno de los sistemas.

Los RAO de cada modo del sistema se calculan entonces mediante la densidad autoespectral de la entrada (elevación de la onda) y la densidad espectral cruzada de la entrada / salida (respuestas del sistema) a través de la siguiente expresión [11]:

$$
\mathrm{RAO}=\frac{\mathrm{S}_{\mathrm{xy}}(\omega)}{\mathrm{S}_{\mathrm{xx}}(\omega)}
$$

donde $\mathrm{S}_{\mathrm{xy}}(\omega)$ y $\mathrm{S}_{\mathrm{xx}}(\omega)$ son las densidades espectral cruzada y autoespectral de la entrada $x(t)$ y la salida $y(t)$, en el dominio de la frecuencia, respectivamente.

Para ello, se ha tenido en cuenta una dirección de incidencia de las olas de cero grados con aire en calma. Además, se han considerado las no linealidades debidas a la flexibilidad de la torre, cargas hidrodinámicas, efecto viscoso y líneas de amarre.

De este modo, se obtienen los RAO de los ocho estados del sistema previamente descritos que se muestran en la Figura $3 a-h$, con un rango de período de RAO entre 2 s y 35 s.

Asimismo, en la Figura 3 se muestran mediante tres curvas en verde, rojo y azul que representan la plataforma de barcaza estándar, la plataforma de barcaza basada en OWC con válvulas abiertas y la

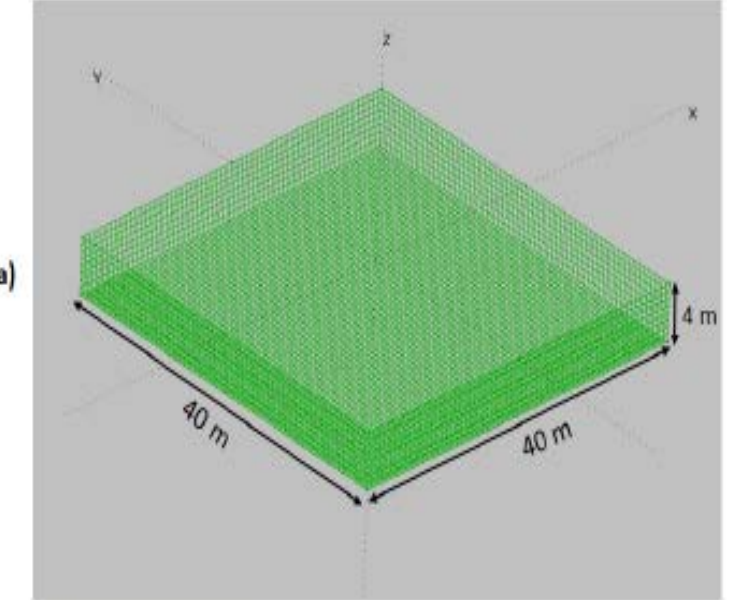

(b)

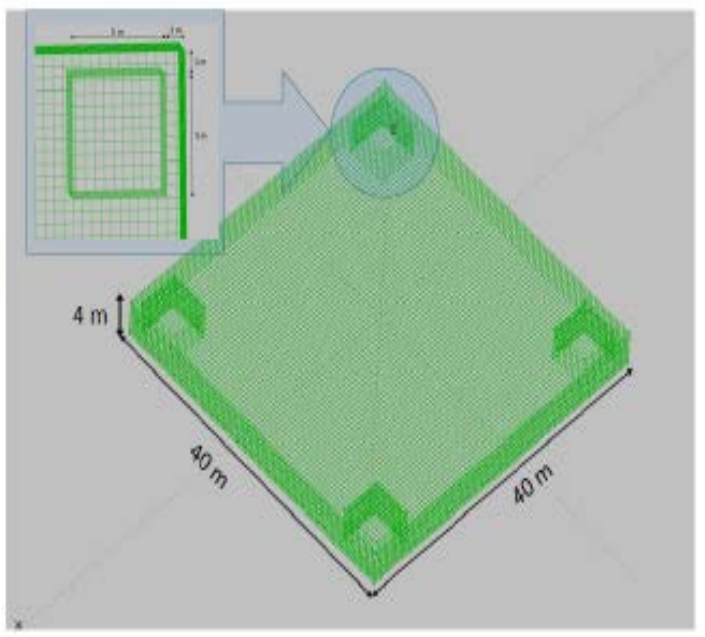

(c)

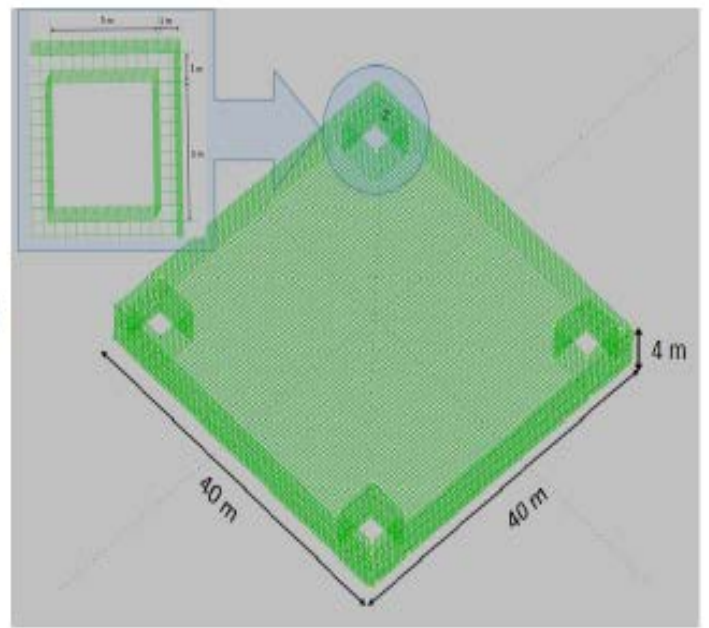

Figura 2. Geometría de plataformas para (a) Plataforma de barcaza estándar. (b) Plataforma de barcazas basada en OWC con válvulas cerradas. (c) Plataforma de barcazas basada en OWC con válvulas abiertas.

plataforma de barcaza basada en OWC con válvulas abiertas, respectivamente. Como cabría esperar, las 

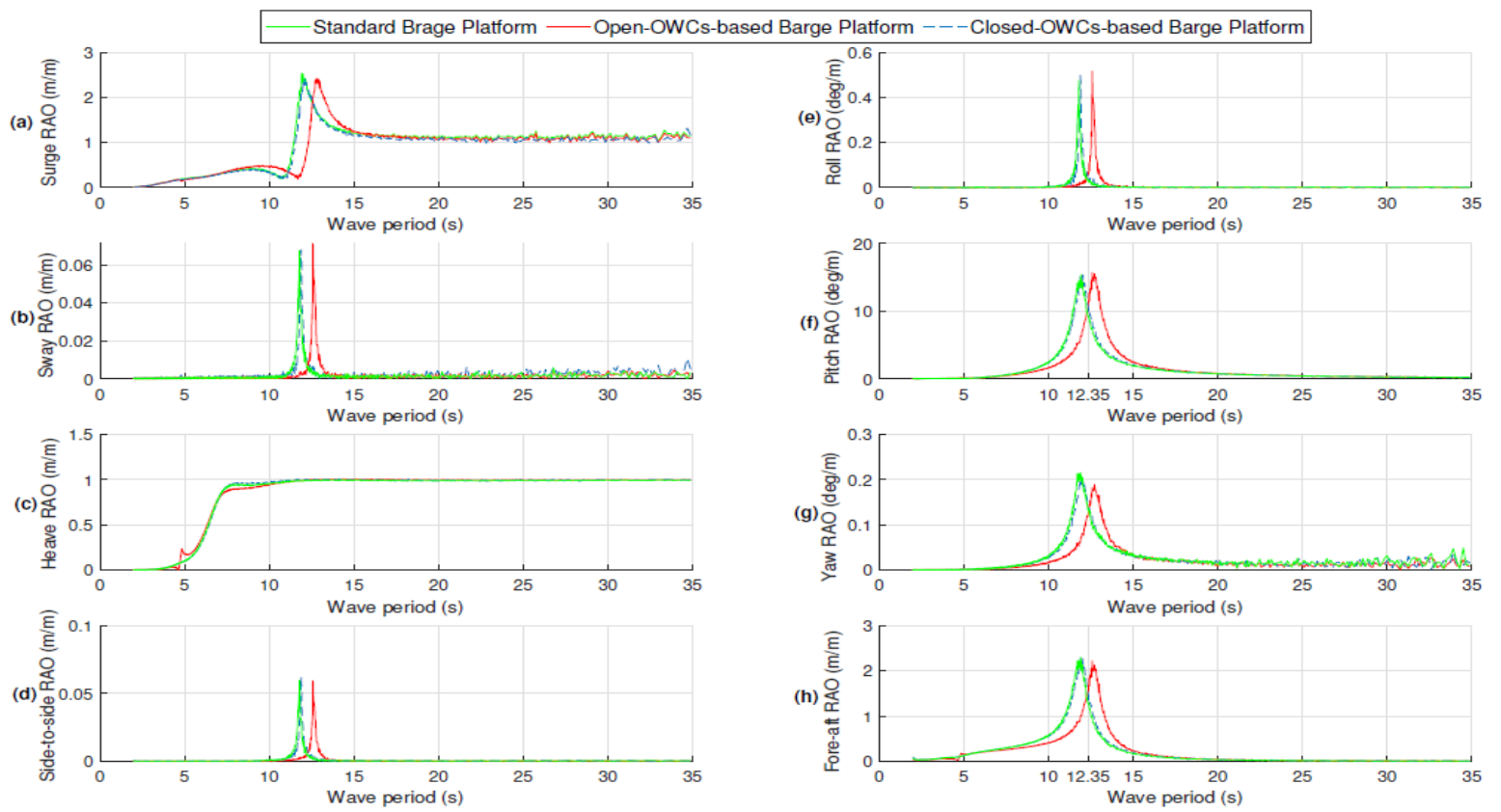

Figura 3. RAO para (a) Desplazamiento en $x$. (b) Desplazamiento en $y$. (c) Elevación. (d) Torre babor-estribor. (e) Balanceo. (f) Cabeceo. (g) Guiñada. (h) Torre proa-popa

curvas de los RAO para la plataforma de barcaza estándar y la plataforma de barcaza basada en OWC cerradas prácticamente idénticas, significando que el comportamiento ambas es el mismo en los diferentes modos.

Además, debido a las condiciones ambientales consideradas, los estados excitados a lo largo de la dirección de la onda incidente presentan oscilaciones muy leves, como se puede observar en los RAO correspondientes de las figuras $3 \mathrm{~b}, \mathrm{~d}$, e, g.

Asimismo, al analizar el RAO de elevación que se muestra en la Figura 3c, se puede ver que las tres topologías presentan curvas RAO similares, lo cual denota que las tres plataformas oscilan de manera similar al elevarse con la ola.

No obstante, el comportamiento más relevante se extrae del análisis de las figuras $3 f$, h, correspondientes al cabeceo (pitch - PRAO) y el cabeceo de la torre, es decir, el desplazamiento proapopa de la misma (fore-aft - FRAO), que da una idea de la estrategia de control propuesta en la Sección 3.2.

Se puede observar además que las curvas PRAO y FRAO para plataformas basadas en barcazas estándar y OWC cerradas son casi idénticas con pequeñas diferencias. Y cuando hay un RAO más alto, la oscilación en ese modo es mayor. Además, los FRAO para las plataformas tienen el mismo comportamiento que los PRAO. Al igual que los PRAO, el FRAO para la plataforma de barcaza abierta con base en OWC es más bajo que la plataforma de barcaza estándar y con plataforma de barcaza cerrada con OWC para períodos inferiores a 12,25 s y 12,35 s respectivamente. Además, para los períodos de más de 12,25 s y 12,35 s, el FRAO de la plataforma de barcaza basada en OWC abiertas es más alto que los FRAO de las plataformas de barcaza estándar y OWC cerradas, respectivamente.

\subsection{Diseño de Control}

En esta sección se propone una estrategia de control de conmutación al objeto de reducir las oscilaciones del sistema, centrando el objetivo en los dos modos que presentan mayor afectación: cabeceo y torre proapopa. Para lo cual se tomarán los valores PRAO Y FRAO, de acuerdo con los resultados del desempeño de las diferentes plataformas ante diferentes estados de la mar obtenidos en la subsección anterior. Así, las figuras $4 a$, b muestran el PRAO y FRAO para las plataformas de barcaza basadas en OWC cerradas y las plataformas de barcaza basadas en OWC abiertas, representadas en azul y rojo, respectivamente.

Puede observarse en la Figura 4a, b que el PRAO y FRAO para la plataforma de barcazas basada en OWCs abiertas tienen valores más bajos en comparación con la plataforma de barcazas basada en OWCs abiertas cuando el período de ola es menor a 12.35 s. Por otro lado, para los períodos de olas superiores a 12,35 s, los PRAO y FRAO para la plataforma de barcaza con base en OWC cerrados son más altos que los de la plataforma de barcaza con base en OWCs abiertas. Esto significa que para períodos de oleaje inferiores a $12,35 \mathrm{~s}$, la plataforma de barcazas basada en OWCs abiertas presenta menor oscilación y por tanto mejor rendimiento que la plataforma de 


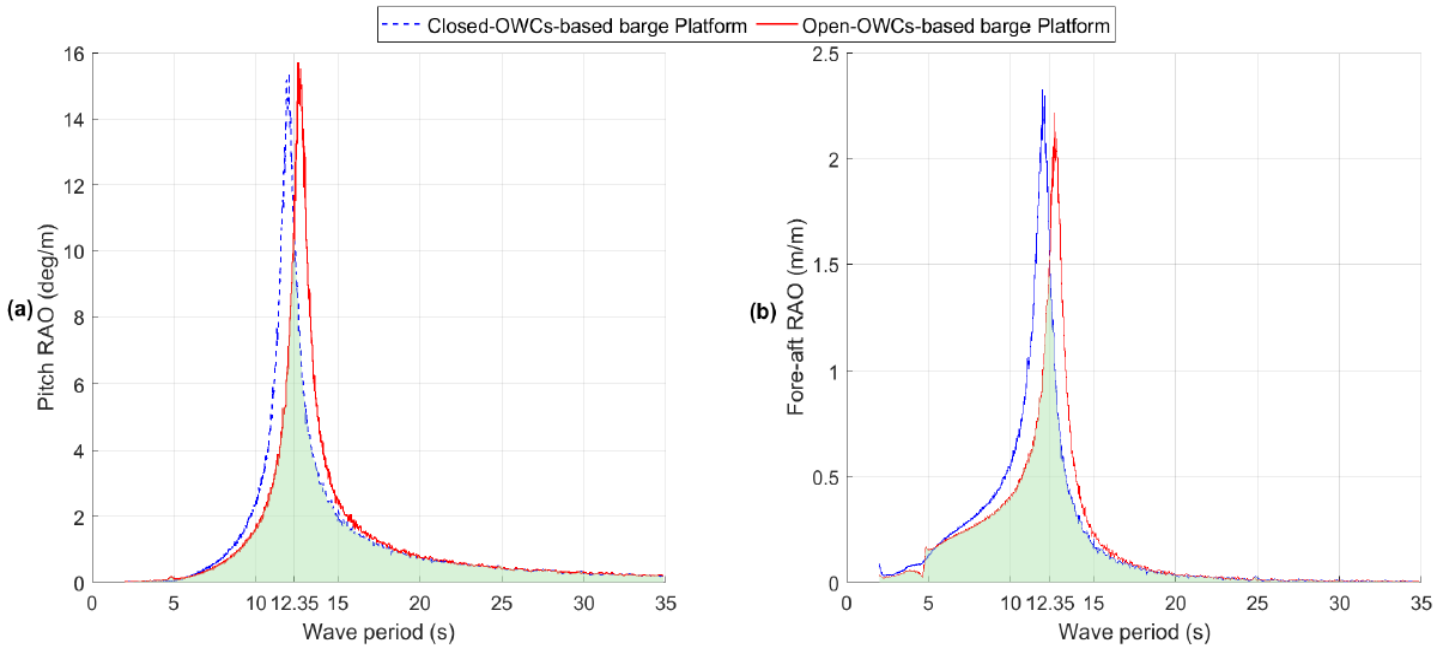

Figura 4. RAO para (a) Cabeceo. (b) Torre proa- popa

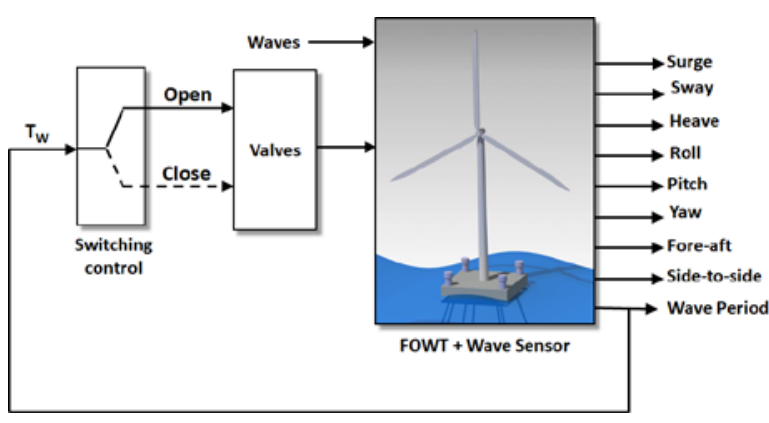

Figura 5. Diagrama de bloques de FWTcontrolado.

barcaza basada en OWCs cerradas. De igual modo, para períodos superiores a 12,35 s, la plataforma de barcaza basada en OWC cerrados presenta una menor oscilación que la plataforma de barcaza basada en OWC abiertos.

De esta forma, es posible establecer regiones, marcadas en verde en la Figura 4a, b, que representan las regiones operativas óptimas con mejor desempeño.

Utilizando estos resultados, se puede establecer el esquema de control de conmutación representado mediante el diagrama de bloques de FWT controlado que se presenta en la Figura 5 y que permite al sistema permanecer en la zona de rendimiento óptimo para cada uno de los modos seleccionados en función del periodo de la ola incidente.

\section{Presentación de Resultados}

Al objeto de mostrar la capacidad del sistema de control propuesto para la estabilización de la plataforma FWT, se han seleccionado dos períodos de onda representativos entre aquellos de mayor y menos duración de $12.35 \mathrm{~s}$, de forma que es posible estudiar el comportamiento de la barcaza controlada basada en OWC y la plataforma de barcaza estándar para diferentes condiciones de estado de la mar. En concreto, se han considerado periodos de ola de $10 \mathrm{~s}$ de 0 a $100 \mathrm{~s}$ con una amplitud de $0,9 \mathrm{~m}$ y de $14 \mathrm{~s}$ a partir de 100 a 240 s con una amplitud de $0.9 \mathrm{~m}$, como se muestra en la Figura 6.

Como se puede ver en las Figuras 3f y 4a, el PRAO para la plataforma de barcaza basada en OWC abiertas es más bajo que el de la plataforma de barcazas estándar durante el período de 10 s. Se puede observar que, consecuentemente, el controlador abre las válvulas OWC de control de flujo, disminuyendo las oscilaciones de cabeceo en un $30.8 \%$ de 4.106 grados para la plataforma de barcaza estándar a 2.841 grados para la plataforma de barcaza controlada basada en OWC, como se muestra en la Figura 7.

Análogamente, se puede ver en las mencionadas Figuras 3f y 4a que, para un período de ola incidente de $14 \mathrm{~s}$, los PRAO para la plataforma de barcaza con base en OWC cerrados son más bajos que en la plataforma de barcaza con base en OWC abiertas. Se pude observar cómo, consecuentemente, después de 100 s, cuando el período de la ola cambia a 14 s en la Figura 7, el controlador cierra las válvulas OWC para reducir la oscilación.

Por su parte, se puede observar que durante el período de $10 \mathrm{~s}$, los FRAO en las Figuras $4 \mathrm{~h}$ y 5b para la plataforma de barcaza basada en OWC abiertas son menores que los de la plataforma de barcazas estándar. De modo que es esperable obtener menores oscilaciones en la torre de proa a popa para el caso de la plataforma de barcaza abierta basada en OWC, en comparación con la plataforma de barcaza estándar. Consecuentemente, como se muestra en la figura 8, el controlador abre las válvulas OWC de $0 \mathrm{~s}$ a $100 \mathrm{~s}$ con período de $10 \mathrm{~s}$. Como resultado, este tipo de oscilaciones disminuyen en un $25 \%$ de $0,9241 \mathrm{~m}$ para la plataforma de barcaza estándar a 0,69 m para la 


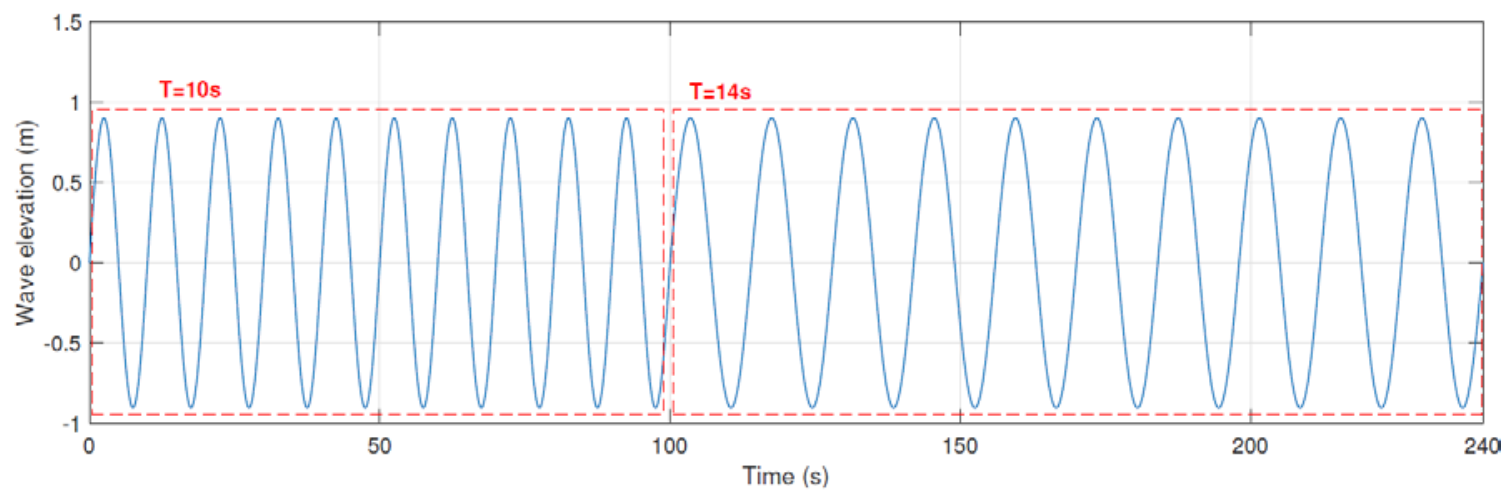

Figura 6. Entrada de ola considerada a las plataformas basadas en barcazas.

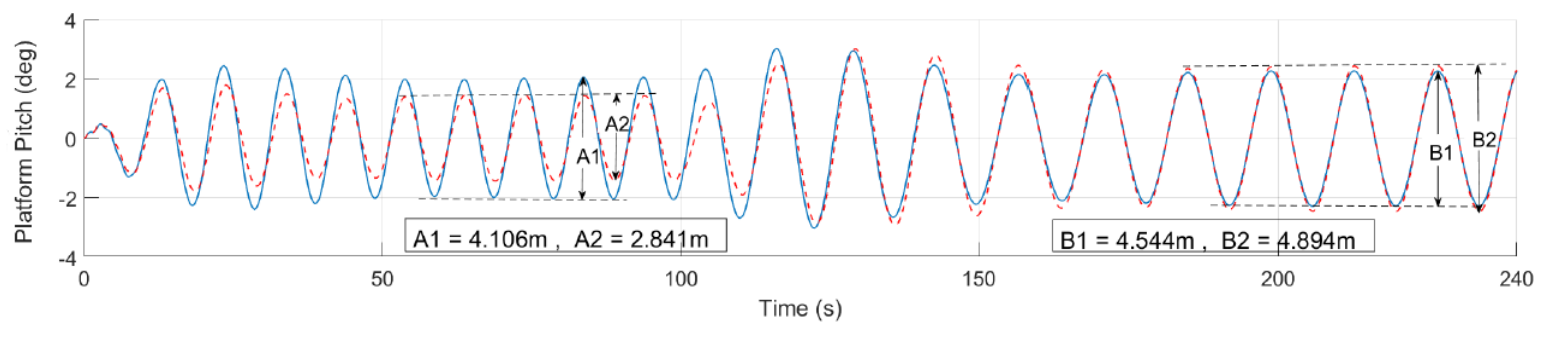

Figura 7. Desplazamientos de pitch

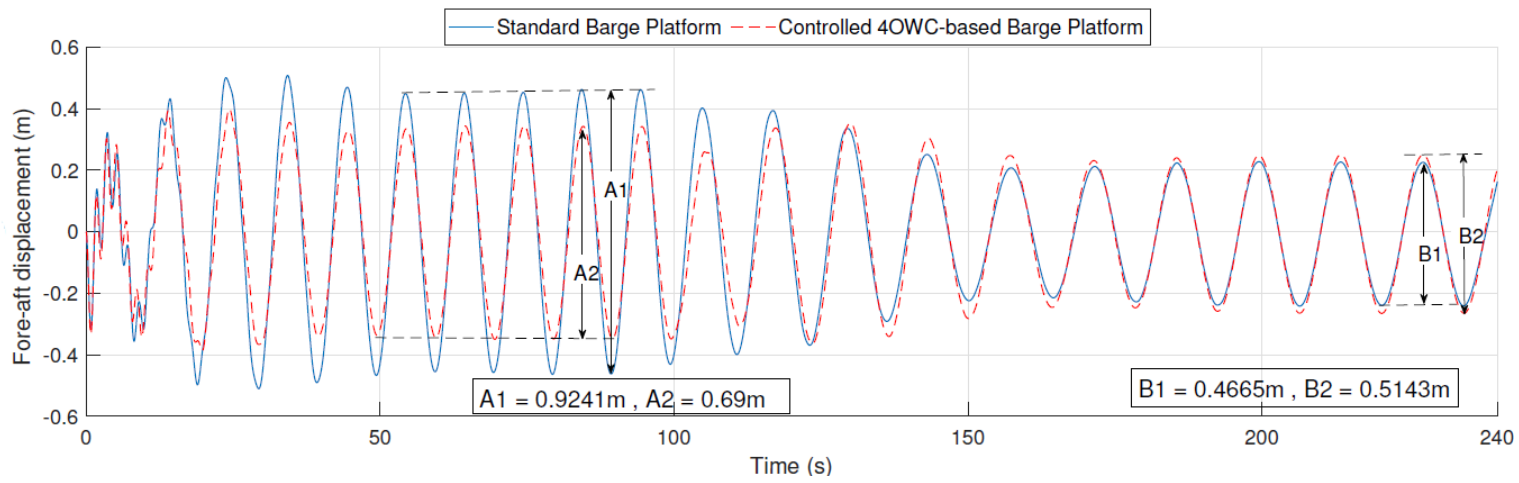

Figura 8. Desplazamientos de proa a popa

plataforma de barcaza controlada basada en OWC, como se muestra la misma Figura 8. De la misma forma, se puede apreciar cómo después de $100 \mathrm{~s}$ el controlador conmuta para cerrar las válvulas OWC, como se corresponde con los FRAO computados en las Figuras 3f y 4a para un período de $14 \mathrm{~s}$.

\section{Conclusiones}

En el presente trabajo se ha propuesto un método de control de conmutación con el fin de disminuir las oscilaciones de sistemas FWT en sus diferentes modos de operación, y en particular cabeceo y desplazamiento proa-popa de la parte superior de la torre. Para ello, se ha analizado la dinámica de los diferentes modos de la FOWT mediante funciones RAO en el dominio de la frecuencia. A partir de los resultados obtenidos, se ha propuesto una nueva estrategia de regulación de las válvulas de control de flujo de aire en los módulos OWC instalados en la plataforma.

Según los RAO, se observó que para los períodos de oleaje más cortos $(<12,35 \mathrm{~s})$, la plataforma de barcazas abierta basada en OWC tiene un mejor rendimiento, por lo que el controlador abre las válvulas de los OWC.

Por otro lado, para los períodos de ola más largos (> $12,35 \mathrm{~s}$ ), el rendimiento de las oscilaciones de la barcaza estándar y las plataformas de barcazas basadas en OWC cerradas es mejor que la plataforma de barcaza basada en OWC abiertas.

Como resultado, el controlador cierra las válvulas de los OWC para reducir efectivamente las oscilaciones en el cabeceo y los movimientos de proa a popa de la torre superior.

$\mathrm{Al}$ objeto de mostrar el desempeño del esquema de control propuesto, se ha considerado un escenario 
representativo con periodos variables de ola incidente. Los resultados muestran que el cabeceo de la plataforma y desplazamiento proa-popa de la torre disminuyen drásticamente para el caso de la plataforma de barcaza controlada basada en OWC, en comparación con la plataforma de barcaza estándar. Es decir, se ha demostrado que la estrategia de control propuesta mejora el rendimiento del sistema al reducir las oscilaciones no deseadas que surgen a frecuencias específicas respecto a plataformas estándar de barcaza no controladas.

\section{Agradecimientos}

Los autores del presente trabajo quisieran agradecer el apoyo del Gobierno Vasco mediante el proyecto IT1207-19 y del MCIU/MINECO a través de los proyectos RTI2018-094902-B-C21 y RTI2018094902-B-C22 (MCIU/AEI/FEDER, UE).

\section{English summary}

\section{A novel Control Strategy for oscillation reduction in Floating Wind Turbines}

\begin{abstract}
In this work, a new control strategy has been proposed to improve the stability of floating wind turbines (FWT). In order to study the reduction of undesired oscillations in the system, and in particular in the platform pitch and fore-aft nacelle movements. A square-shaped barge-type platform has been considered and equipped with four symmetrically located Oscillating Water Columns (OWCs). In this way, the air flow control valves of the capture chambers allow the aforementioned air columns to be operated so that to control the movements of the barge caused by the oscillatory dynamics of the incident wave. To do so, an analysis of the response amplitude operators (RAO) has been carried out, which in turn allows the implementation of a new switching control strategy to properly regulate the transition of the flow control valves. The results obtained show that the proposed hybrid platform topology, equipped with OWC controlled systems, presents better performance than a traditional analog platform.
\end{abstract}

Keywords: floating wind turbine; oscillating water column; wave energy; wind power; stabilization; response amplitude operator; switching control.

\section{Referencias}

[1] Karimirad, M., Koushan, K, (2016) "WindWEC: Combining wind and wave energy inspired by hywind and wavestar", In Proceedings of the 2016 IEEE International Conference on
Renewable Energy Research and Applications (ICRERA), Birmingham, UK, pp. 96-101.

[2] Maria-Arenas, A., Garrido, A.J., Rusu, E., Garrido, I., (2019) "Control strategies applied to wave energy converters: State of the art”, Energies, p. 3115.

[3] Lackner, M.A., (2013) “An investigation of variable power collective pitch control for load mitigation of floating offshore wind turbines”, Wind Energy, pp. 435-444.

[4] Matha, D., (2009) "Model Development and Loads Analysis of an Offshore Wind Turbine on a Tension Leg Platform with a Comparison to Other Floating Turbine Concepts”, National Renewable Energy Lab. (NREL): Golden, CO, USA.

[5] Slocum, A., Kluger, J., Mannai, S., (2019) "WindWEC: Combining wind and wave energy inspired by hywind and wavestar", In Proceedings of the 2019 Offshore Energy and Storage Summit (OSES), Brest, France, pp. 1-6.

[6] Kamarlouei, M., Gaspar, J.F., Calvario, M., Hallak, T.S., Mendes, M.J., K, Thiebaut, F., Soares, C.G. (2020) "Experimental analysis of wave energy converters concentrically attached on a floating offshore platform”, Energy, pp. 1171-1185.

[7] Zhang, Y., Zhao, X., Wei, X. (2020) "Robust structural control of an underactuated floating wind turbine”, Wind Energy, pp. 2166-2185.

[8] Hu, Y., Wang, J., Chen, M.Z., Li, Z., Sun, Y, (2018) "Load mitigation for a barge-type floating offshore wind turbine via inerter-based passive structural control”, Eng. Struct, pp. 198-209.

[9] Jonkman, J.M. (2007) "Dynamics Modeling and Loads Analysis of an Offshore Floating Wind Turbine”, National Renewable Energy Lab. (NREL): Golden, CO, USA.

[10] Aboutalebi, P., M’zoughi, F., Garrido, I., Garrido, A.J., (2021) "Performance Analysis on the Use of Oscillating Water Column in Barge-Based Floating Offshore Wind Turbines”, Mathematics, p. 475.

[11] Ramachandran, G.K.V., Robertson, A., Jonkman, J.M., Masciola, M.D, (2013) "Investigation of Response Amplitude Operators for Floating Offshore Wind Turbines", National Renewable Energy Lab. (NREL): Golden, CO, USA. 